International Journal of Ophthalmology \& Eye Science (IJOES)

ISSN 2332-290X

\title{
Exfoliation Syndrome
}

Madonna $\mathrm{RJ}^{1 *}$, Gould $\mathrm{J}^{2}$

${ }^{1}$ SUNY College of Optometry, New York, NY, USA.

${ }^{2}$ State University of New York College of Optometry, New York, USA.

\section{*Corresponding Author:}

Richard J. Madonna, MA, OD, FAAO,

SUNY College of Optometry, 33 West 42nd Street New York, NY, USA.

E-mail: rmadonna@sunyopt.edu

Received: March 15, 2015

Accepted: March 25, 2015

Published: March 26, 2015

Citation: Madonna RJ, Gould J (2015) Exfoliation Syndrome. Int J Ophthalmol Eye Res. S1:005, 23-26. doi: http://dx.doi.org/10.19070/2332290X-SI01005

Copyright: Madonna RJ ${ }^{\circ}$ 2015. This is an open-access article distributed under the terms of the Creative Commons Attribution License, which permits unrestricted use, distribution and reproduction in any medium, provided the original author and source are credited.

\section{Exfoliation Syndrome}

The age-related systemic condition called pseudoexfoliation syndrome or exfoliation syndrome underlies a myriad of ocular findings and abnormalities. The best known relationship is to glaucoma; however, all parts of the eye may be affected. The link to systemic diseases is not as clear despite the systemic nature of the condition. Recently, genetics and environmental factors have been found to play a role in its development.

\section{Terminology}

The condition was first identified in 1917 by the Finnish ophthalmologist John G. Lindbergh who described whitish material on the lens capsule and pupillary border [1]. The term pseudoexfoliation syndrome (PEX, PXE, PES, PXF. XFS) was used because the condition was similar in appearance to true exfoliation (peeling) of the lens capsule that occurred as a result of excessive infrared radiation or heat exposure to the crystalline lens of glassblowers [2]. With the widespread use of protective measures to reduce infrared and heat exposure and resultant reduction in prevalence of true exfoliation, the pseudo- is now being dropped and the term exfoliation syndrome (ES) is taking hold in the literature. Following suit, we will use this term along with exfoliation glaucoma (EG) rather than pseudoexfoliation glaucoma in this review. However, the reader should understand that the terms pseudoexfoliation and exfoliation are still used interchangeably.

\section{Epidemiology}

Historically, ES has been thought to be mostly associated with people of Scandinavian descent but it is now clear that ES occurs across all races and ethnicities with differing prevalence in specific populations and geographic areas. In some Scandinavian countries, the prevalence of ES may be greater than $20 \%$ in persons over age 60 [3] but it is also highly prevalent in populations as diverse as Greeks [4], in the Bantu tribesman in South Africa [5], the Inuit population [6], in Australian aborigines [7] and in South India [8]. ES appears to be uncommon in those of Chinese descent [9] and occurs in differing degrees across Africa [10].

Strong relationships have been shown with increasing age and the development of ES [11, 12]. Although studies looking at prevalence among gender have varied widely, most evidence indicates that female gender is associated with an increased risk of ES [13].

\section{Genetics and Environmental Factors}

In Caucasians, two different single-nucleotide polymorphisms (SNPs) of the lysyl oxidase-like 1 (LOXL-1) gene on chromosome 15 have been shown to be related to ES [3, 14]. LOXL-1 is associated with the formation and maintenance of elastic tissue. These SNPs occur in nearly identical frequency in populations with markedly diverse prevalence of ES $[3,15]$ suggesting the likelihood of other factors modifying the expression of these gene variants. ES is known to occur at higher prevalence with increased latitude in populations throughout Europe, the Middle East, Asia and in the United States [13]. It is reported that living in the southern tier of the United States is associated with a marked reduction in risk for both ES and EG as compared with living in the northern tier of the United States. Numbers of days of sunshine and average temperature appear to be at least in part major determinants of the expression of the gene $[13,16]$.

A number of other gene variants have been found to be associated with both ES and EG; their significance is now being investigated $[17,18]$.

\section{Pseudoexfoliation Material Composition and Production}

Exfoliative material (EM) is actively produced by numerous cell types throughout the body. Within the eye, the corneal endothelium, pre-equatorial lens epithelium, non-pigmented ciliary epithelium, trabecular endothelial cells and iris epithelial cells have all been shown to produce EM [19]. Systemically, the EM is usually found in areas high in connective tissue and specifically from connective tissue fibroblasts, smooth and striated muscle tissue and cardiomyocytes $[11,20]$. 
While the exact pathophysiology is not completely known, it appears that EM is a fibrillar extracellular material that progressively deposits itself onto intraocular and extraocular tissues. Extensive investigation has revealed multiple important components of the material. The fibrillar extracellular matrix is composed of a highly cross-linked combination of glycoproteins and proteoglycans. Using immunohistochemistry, the exfoliation fibrils are shown to contain primarily fibrillin-1 but other epitopes of the elastic fiber system have also been identified in smaller amounts. It is theorized that EM is a result of excessive production and aggregation of elastic microfibrillar components [19]. Components of the basement membrane system (i.e. fibronectin, laminin, etc.) are also present within EM, thus suggesting its production is a result of abnormal basement membrane metabolism [19]. Active LOXL-1, which serves as an extracellular chaperone for clusterin in addition to its role in elastic fiber homeostasis, has also been found within EM in early ES [21, 22]. The presence of active LOXL-1 suggests involvement in the abnormal cross-linking and improper protein folding leading to the aggregation and accumulation of EM. In late stages of ES/EG, LOXL-1 expression is down-regulated which may lead to insufficiency in cross linking and elastic matrix alterations contributing to glaucomatous damage and progression [19].

An increased expression of matrix metalloproteinase (MMP)-2 and tissue inhibitors of matrix metalloproteinase (TIMP)-1 and TIMP-2 is evident in cells that produce EM [19]. The upregulation of TIMP-1 and TIMP-2 are more pronounced than the upregulation of MMP-2. This imbalance contributes to a low grade inflammatory response, increased oxidative stress and accumulation of EM which can play a role in ES/EG development [19].

\section{Systemic Manifestations of Exfoliation Syndrome}

While the target tissues of greatest significance appear to be in the eye, ES has been found throughout the body. Using electron microscopy and light microscopic immunohistochemistry, ES has been found in the heart, lungs, liver, kidney, gallbladder and cerebral meninges of patients with ocular ES $[11,23]$. Patients with ES have elevated levels of homocysteine which is a well-known risk factor for cardiovascular disease [24, 25]. Systemic associations have been found between ES and hypertension, myocardial infarction, stroke, transit ischemic attacks, Alzheimer's disease and sensorineural hearing loss [23].

\section{Ocular Manifestations of Exfoliation Syndrome}

Ocular manifestations of ES appear unilaterally in 50\%-75\% of patients with about $1 / 3$ more developing the condition in the fellow eye within 10 years [26]. As expected with a systemic disease, ultrastructural examination has shown that patients with a unilateral clinical presentation are actually affected bilaterally [27, 28]. The ocular findings in ES are diverse and affect the entire eye, leading to a broad range of ocular complications.

The clinical diagnosis of ES is usually made by the discovery of EM on the pupillary margin and anterior lens capsule where it appears as a white, fluffy, dandruffy-like material. The EM often has a patchy distribution along the pupillary ruff. Upon pupillary dilation, the anterior lens capsule classically contains three distinct zones: 1) a circular central disc of EM; 2) a surrounding area of clear capsule surface where EM has been rubbed off because of capsular contact with the iris; and 3) a peripheral deposition of EM with a granular appearance (Figure 1). Lenticular contact with the iris causes liberation of pigment from the posterior iris epithelial cells. Careful examination of the sphincter will reveal atrophy with irregular areas of transillumination; this is often referred to as a moth-eaten appearance of the iris sphincter (Figure 2). The transillumination defects can be distinguished from those seen in pigment dispersion syndrome (PDS) because of their irregular appearance and location closer to the pupil versus the regular mid-peripheral defects seen in PDS. Liberated pigment from the posterior iris epithelium is deposited onto the corneal endothelium and into the anterior chamber angle. On the cornea, the deposition can occasionally take the form of a Krukenberg's spindle as seen in PDS. Within the angle, the pigmentation is distinguished from that of PDS in that the pigment seen in ES is patchy with the highest density appearing in the inferior angle as opposed to the 360 degree band of pigmentation in PDS. A Sampaolesi line is often present. Its appearance may precede EMdeposition on the anterior lens and other pigmentary signs of ES [11].

Figure 1. The classic appearance of exfoliative material deposited on the anterior lens capsule with inner and outer rings of EM deposition with a central clear zone

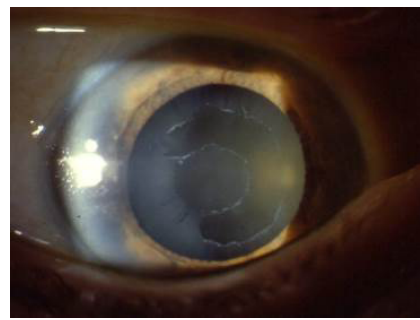

Figure 2. Irregular iris transillumination defects near to the pupil in a patient with ES.

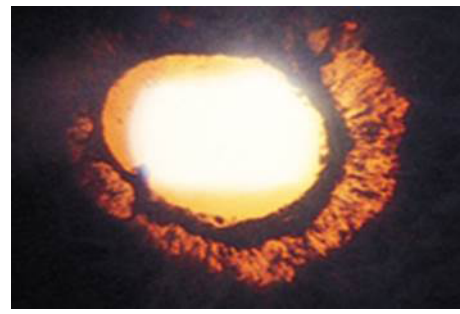


Early cataract formation is common $[11,29]$. Poor pupillary dilation due to iris sphincter hypoxia, weakened zonular attachments with lens or IOL dislocation and an increased risk of capsular tears make cataract surgery more problematic for the surgeon. Careful pre-operative examination to recognize phacodonesis is important to identify those with weak zonules. IOP spikes, significant anterior chamber inflammation, corneal edema and capsular opacification are more common in the post-operative period.

The deposition of ESM on other ocular structures may also lead to complications. A breakdown in the blood-aqueous barrier may result in low-grade anterior chamber flare and subsequent posterior synechiae formation.

Persons with ES are more likely to have narrow angles with an increased risk of angle closure because of zonular laxity and anterior migration of the lens [30].

EM development is closely related to vascular tissues, as previously discussed. As such, an increased risk of venous occlusions and optic disc hemorrhages has also been reported in patients with ES [11].

\section{Exfoliative Glaucoma}

ES is the most common identifiable cause of glaucoma worldwide. The risk of developing glaucoma is cumulative with time, echoing the increased prevalence of ES with age. Glaucoma is 5-10 times more common is ES patients than in those without exfoliation.

Approximately $25 \%$ of ES patients have elevated IOP and one third of these have glaucoma. It is estimated that about 60 to 70 million people worldwide have ES so about 5 to 6 million persons have EG. This is about $10 \%$ of the world's glaucoma [11].

Elevated IOP in ES is due to a reduction in outflow facility in both the trabecular and uveoscleral outflow systems [31] presumably from deposition of EM and subsequent ultrastructural changes in the outflow pathways. It is believed that the pigment deposition in ES contributes to the reduction in outflow as well. IOP elevation is correlated with both the amount of EM and pigment released [32].

While EG is commonly thought of as a high-pressure glaucoma, evidence of increased optic nerve susceptibility to damage via vascular or laminar routes indicates that the more involved eye may have a greater risk of developing glaucomatous damage even in the presence of equal IOPs [33]. The previously described variants in the LOXL-1 gene may play a role because of its function as an important enzyme in the formation of elastin [11].

ES may also underlie an increased risk of developing angle-closure glaucoma (ACG). A combination of anterior lens movement due to zonular weakness, posterior synechiae formation and increased iris thickness are proposed mechanisms for this increased risk [30].

Exfoliative glaucoma is more severe than primary open-angle glaucoma by any measure. Patients have higher IOP, are more likely to convert from ocular hypertension to glaucoma, are more likely to have damage at presentation, do not respond as well to treatment, have more frequent and higher spikes in IOP, and are more likely to progress regardless of the level of IOP [34]. In the Early Manifest Glaucoma Trial, untreated EG patients progressed at a rate of $3.13 \mathrm{~dB} /$ year as compared to untreated POAG patients who progressed at a rate of $1.31 \mathrm{~dB} /$ year, despite nearly identical entering IOPs. Additionally, the EG group was much more likely to have extremely high rates of progression, with the highest being $11.33 \mathrm{~dB}$ per year. The median time to progression was 19.5 months in the EG group and 44.8 months for the POAG group. Finally, in multivariate analysis, having ES increased the hazard ratio of progressing by more than double as compared to not having ES. The presence of ES was the strongest baseline factor predicting progression in this study [35].

Treatment of EG is similar to POAG but treatment response is less predictable. As with POAG, prostaglandin analogs are usually the drug of first-choice unless contraindicated. Adjunctive medical therapy is also consistent with POAG therapy. EG patients will not respond as well to treatment and will need more adjunctive therapy sooner as compared to POAG patients.

Miotic therapy should be considered in EG patients. Miotics help clear the aqueous and stabilize the pupil so that less exfoliative material is available to deposit in the meshwork. Pilocarpine $2 \%$ qid or less often or slow-release pilocarpine ointment have been utilized. It should be noted, however, that miotics may aggravate the blood-aqueous dysfunction found in ES and also increase the risk of posterior synechiae and cataract formation [36].

Laser trabeculoplasty (ALT or SLT) has proven to be a very effective treatment in EG. This is not unexpected as these patients have greater amounts of pigment in the anterior chamber angle. However, as with medical therapy, IOP control may be lost earlier and there is a greater risk of treatment failure in patients receiving LTP [37].

Because of the higher IOP and greater morbidity in patients with EG, these patients may go on to glaucoma surgery earlier than POAG patients. Trabeculectomy results are comparable [11]. Newer surgical approaches and combination procedures are currently being investigated $[38,39]$.

\section{Conclusion}

The systemic condition ES has its best known and greatest effect in the eye. The glaucoma associated with ES is aggressive and difficult to treat. Early identification may be able to reduce the morbidity associated with it. Genetic and environmental influences have been recently identified that may allow early identification and reduction in ocular morbidity.

\section{References}

[1]. Tarkkanen A, Kivela T, John G (2002) Lindberg and the discovery of exfoliation syndrome. Acta Ophthalmol 80:151-4.

[2]. Dvorak-Theobald G.D (1954) Pseudo-exfoliation of the lens capsule. Am J Ophthalmol 37:1-12.

[3]. Thorleifsson G, Magnusson KP, Sulem P (2007) Common sequence variants in the LOXL1 gene confer susceptibility to exfoliation glaucoma. Science 317:1397-1400.

[4]. Topouzis F, Wilson MR, Harris A (2007) Prevalence of open angle glaucoma in Greece: the Thessaloniki Eye Study. Am J Ophthalmol 144:511-9.

[5]. Bartholomew RS (1973) Pseudocapsular exfoliation in the Bantu of South 
Africa.II. Occurrence and prevalence. Br J Ophthalmol 57: 41-5.

[6]. Ostenfeld-Akerblom A (1988) Pseudoexfoliation in Eskimos (Inuit) in Greenland. Acta Ophthalmol 66:467-8.

[7]. Taylor HR, Hollows FC, Moran D (1977) Pseudoexfoliation of the lens in Australian aborigines. Br J Ophthalmol 61: 473-5.

[8]. Krishnidas R, Nirmalan PK, Ramakrishnan R (2003) Pseudoexfoliation in a rural population of southern India: the Aravind Comprehensive Eye Survey. Am J Ophthalmol 135: 830-7.

[9]. Young AL, Tang WW, Lam DS (2004) The prevalence of pseudoexfoliation syndrome in Chinese people. Br J Ophthalmol 88:193-5.

[10]. Olawoye OO, Pasquale LR, Ritch R (2014) Exfoliation syndrome in subSaharan Africa. Int Ophthalmol 34:1165-73.

[11]. Ritch R (2014) Ocular and systemic manifestations of exfoliation syndrome. J Glaucoma 23: S1-S8.

[12]. Ringvold A (1999) Epidemiology of the pseudoexfoliation syndrome. Acta Ophthalmol 77: 371-375.

[13]. Kang JH, Loomis S, Wiggs J, Stein J, Pasquale LR (2012) Demographics and geographic features of exfoliation glaucoma in two United States-based prospective cohorts. Ophthalmol 119:27-35.

[14]. Hewitt AW, Sharma S, Burdon KP, Wang JJ, Baird PN, et al. (2008) Ancestral LOXL1 variants are associated with pseudoexfoliation in Caucasian Australians but with markedly lower penetrance than in Nordic people. Hum Mol Gen 17:710-16.

[15]. McCarty CA, Taylor HR (2000) Pseudoexfoliation syndrome in Australian adults. Am J Ophthalmol 129:629-33.

[16]. Stein JD, Pasquale LR, Talwar N (2011) Geographic and climatic factors associated with exfoliation syndrome. Arch Ophthalmol 129:1053-60.

[17]. Nakano M, Ikeda Y, Tokuda Y (2014) Novel common variants and susceptible haplotype for exfoliation glaucoma specific to Asian population. Sci Rep 4:5340.

[18]. Ayub H, Micheal S, Akhtar F (2014) Association of a polymorphism in the BIRC6 gene with pseudoexfoliative glaucoma. PLoS One 9(8):e105023.

[19]. Zenkel M, Schlötzer-Schrehardt U (2014) The composition of exfoliation material and the cells involved in its production. J Glaucoma 23:S12-S14.

[20]. Schlötzer-Schrehardt U, Naumann GOH (2006) Ocular and systemic pseudoexfoliation syndrome. Am J of Ophthalmol 141: 921-37.

[21]. Schlotzer-Schrehardt U, Pasutto F, Sommer P (2008) Genotype-correlated expression of lysyl oxidase-like 1 in ocular tissues of patients with pseudoexfoliation syndrome/glaucoma and normal patients. Am J Pathol 173:172435.

[22]. Zenkel M, Kruse FE, Ju“ nemann AG (2006) Clusterin deficiency in eyes with pseudoexfoliation syndrome may be implicated in the aggregation and deposition of pseudoexfoliative material. Invest Ophthalmol Vis Sci 47:1982-90.

[23]. Naumann GO, Schlötzer-Schrehardt U, Küchle M (1998) Pseudoexfoliation syndrome for the comprehensive ophthalmologist.Intraocular and systemic manifestations. Phthalmology 105: 951-68.

[24]. Roedl JB, Bleich S, Reulbach U (2007) Homocysteine in tear fluid of patients with pseudoexfoliation glaucoma. J Glaucoma 16:234-9.

[25]. Clement CI, Goldberg I, Healey PR, Graham SL (2009) Plasma homocysteine, MTHFR gene mutation, and open-angle glaucoma. J Glaucoma
18:73-8.

[26]. Henry JC, Krupin T, Schmitt M (1987) Long-term follow-up of pseudoexfoliation and the development of elevated intraocular pressure. Ophthalmol 94:545-52.

[27]. Hammer T, Schlötzer-Schrehardt U, NaumannGO (2001) Unilateral or asymmetric pseudoexfoliation syndrome? An ultrastructural study. Arch Ophthalmol 119:1023-31.

[28]. Parekh P, Green WR, Stark WJ, Akpek EK (2008) Electron microscopic investigation of the lens capsule and conjunctival tissues in individuals with clinically unilateral pseudoexfoliation syndrome. Ophthalmology 115:61419.

[29]. Kanthan GL, Mitchell P, Burlutsky G (2013) Pseudoexfoliation syndrome and the long-term incidence of cataract and cataract Surgery: The Blue Mountains Eye Study. Am J Ophthalmol 155:83-88.

[30]. Ritch R (1994) Exfoliation syndrome: clinical findings and occurrence in patients with occludable angles. Trans Am Ophthalmol Soc 92:845-944.

[31]. Gharagozloo NZ, Baker R, Brubaker RF (1992) Aqueous dynamics in exfoliation syndrome. Am J Ophthalmol 114:473-78.

[32]. Schlotzer-Schrehardt U, Naumann GOH (1995) Trabecular meshwork in pseudoexfoliation syndrome with and without openangle glaucoma.A morphometric, ultrastructural study. Invest Ophthalmol Vis Sci 36:1750-64.

[33]. Netland PA, Ye H, Streeten BW (1995) Elastosis of the lamina cribrosa in pseudoexfoliation syndrome with glaucoma. Ophthalmology 102:878-86.

[34]. Ritch R, Schlotzer-Schrehardt U (2001) Exfoliation syndrome. Surv Ophthalmol 45:265-315.

[35]. Heijl A, Bengtsson B, Hyman L, Leske MC (2009) Early Manifest Glaucoma Trial Group.Natural history of open-angle glaucoma. Ophthalmology 116:2271-6.

[36]. Angelilli A, Ritch R (2009) Directed therapy: an approach to the improved treatment of exfoliation syndrome. Middle East Afr J Ophthalmol 16:35-40.

[37]. Ayala M1, Chen E (2011) Comparison of selective laser trabeculoplasty (SLT) in primary open angle glaucoma and pseudoexfoliation glaucoma. ClinOphthalmol 5:1469-73.

[38]. Widder RA, Dinslage S, Rosentreter A (2014) A new surgical triple procedure in pseudoexfoliation glaucoma using cataract surgery, Trabectome, and trabecular aspiration. Graefes Arch ClinExpOphthalmol 252: 1971-5.

[39]. Buchacra O, Duch S, Milla E, Stirbu O (2011) One-year analysis of the iStent trabecular microbypass in secondary glaucoma. Clin Ophthalmic 5: $321-6$.

Special Issue on

"Glaucoma and Hypertensive Retinopathy"

Edited by:

Dr. Pinakin Gunvant Davey, Western University of Health Sciences, USA

E-mail:pdavey@westernu.edu 